\title{
Dielectric constant measurement of low loss liquids using stacked multi ring resonator
}

\author{
ANAGHA A KUNTE ${ }^{1,2, *}$ and ARUN N GAIKWAD ${ }^{3}$ \\ ${ }^{1}$ Department of Electronics and Telecommunication, Sinhgad College of Engineering, Pune 411041, India \\ ${ }^{2}$ Department of Electronics and Telecommunication, Marathwada Mitra Mandal's College of Engineering, \\ Pune 411052, India \\ ${ }^{3}$ Department of Electronics and Telecommunication, Zeal College of Engineering and Research, Pune 411041, \\ India \\ e-mail: anaghakunte7@gmail.com; arungkwd47@gmail.com
}

MS received 16 December 2017; revised 11 June 2018; accepted 5 July 2018; published online 3 December 2018

\begin{abstract}
The concept of dielectric constant measurement has been extended and applied in agriculture, pharmaceutical and food industry for quality control of liquids. Dielectric analysis of material at microwave frequencies can be done using novel shielded stacked multi-ring resonator (SMRR). The dielectric constant of liquids and paste has been calculated using SMRR with greater accuracy than the planar resonator, boxed resonator and stacked resonator. SMRR contains a ring resonator with fed patch and parasitic patch with different numbers and sizes of rings. The dimensions of rings on the parasitic patch are optimized to achieve Quality factor Q greater than 100 and return loss less than $-2 \mathrm{~dB}$. Due to dual resonance in novel SMRR, structure losses are reduced by $50 \%$ than planar resonator structure. The behavior of SMRR structure at the $2.45 \mathrm{GHz}$ frequency is studied with $\mathrm{E}$ field and $\mathrm{H}$ field. 3D model is designed in Computer Simulation Technology Microwave Studio (CST MWS) using TLM (Transmission Line Modeling) solver. Electromagnetic field analysis as well as impedance bandwidth of SMRR using CST MWS 3D model prove that electromagnetic coupling in SMRR structure increases thus improves quality factor. In SMRR quality factor increases and losses reduce help us to predict the complex permittivity of material for quality analysis.
\end{abstract}

Keywords. Stacked multi ring resonator; dielectric constant; quality factor; return loss.

\section{Introduction}

The dielectric constant of liquids is necessary to find adulteration in food, agriculture, pharmaceutical and petroleum industry. There are always better and newer techniques which are upcoming in the market for determining dielectric constant. Various techniques have been developed like microwave resonant techniques, open-ended waveguide technique, resonant cavity, etc. These nondestructive resonant techniques are based on reflection and transmission coefficient measurements. One of the techniques is microstrip ring resonator $[1,2]$ which can be used for dielectric constant measurement. [3] Resonant method can be applied to low loss material as this is a narrow bandwidth method. To evaluate low loss material microstrip resonator can be used. The newly developed structure of SMRR can be utilized for complex permittivity measurement of liquids and paste. The objective of this paper is to enhance the accuracy of the stacked resonator by unique

*For correspondence placement of multiple rings on the parasitic patch. The SMRR structure consists of the fed patch and parasitic patch. It is also simpler in construction. It is easier to handle liquid material and measure dielectric constant compared to those from the literature.

The tested liquid like N-Hexane, diesel, wax, etc. have the low permittivity and loss, long relaxation time and a broad frequency dispersion region. Hence, the permittivity spectrum has to be measured with high sensitivity over a broad frequency range (typically $1 \mathrm{kHz}$ to $10 \mathrm{GHz}$ ), which requires sensitive measurement cells and adequate models for calculating the permittivity [4]. Thus to fulfil the said requirements, we have designed a novel SMRR for complex permittivity measurement in the frequency domain. The SMRR consists of the lower patch of the ring called as the fed patch which is excited by resonating frequency of $2.45 \mathrm{GHz}$, and the upper patch is known as the parasitic patch.The tested liquid fills the partial space inside the SMRR.

The geometry of shielded ring resonator is as discussed in [5]. RT Duroid 5880 substrate is used for the fed patch. 
Formulae used for designing are as specified in [6-8]. When the size of the parasitic patch is nearly equal to the fed patch and the spacing between the fed patch and the parasitic patch is kept about $0.45 \lambda<\lambda<0.5 \lambda$, maximum energy coupling is obtained [9]. The accurate near field analysis by the Transmission Line Matrix (TLM) computational electromagnetic method makes it clear that the space between the fed patch and the parasitic patch forms the resonator. TLM solver can be used for dielectric characterization of material [10].

When the higher gain is obtained, the amplitude of electric fields in the cavity between patches becomes large. The amplitude of current distribution on the parasitic patch also becomes large [9]. Due to higher $\mathrm{Q}$ value and low insertion loss, determination of relative permittivity of material under test with the developed model is highly accurate [11].

CST MWS allows better handling of design with electromagnetic coupling between different parts of the SMRR structure. Simulated parameters like conductor loss, dielectric loss, effective dielectric constant, line impedance, power lost in dielectrics and metals are studied for investigation of near field distribution in SMRR. S-parameters, electric field, magnetic field, surface current distributions are computed to study electromagnetic field inside the SMRR. The measured values of insertion loss $S_{21}$, resonating frequency $f r$ and loaded quality factor $Q_{L}$ are used to analyze reduction of losses. The development of a prototype boxed microstrip resonator was considered as the reference [12]. Planar ring resonator, boxed ring resonator, stacked ring resonator and SMRR structures are developed and optimized for dielectric constant measurement.

\section{Structures of resonator}

The various structures of the ring resonator i.e., planar, boxed and stacked ring resonator are shown in figures 1, 2 and 3, respectively. The suggested SMRR structure is an enhancement of stacked ring resonator structure [5] and

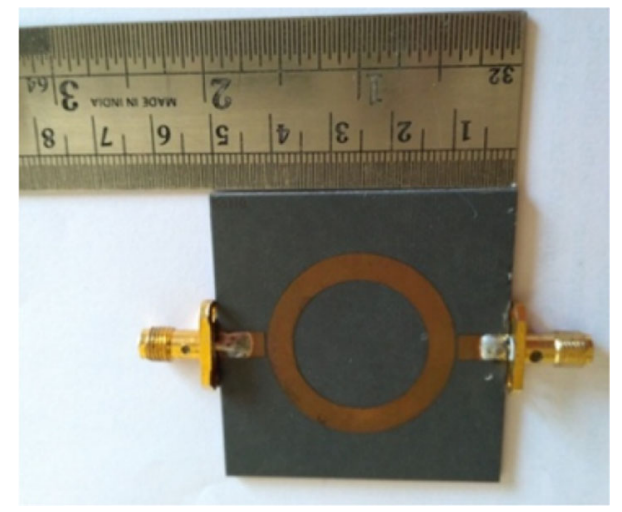

Figure 1. Planar resonator.

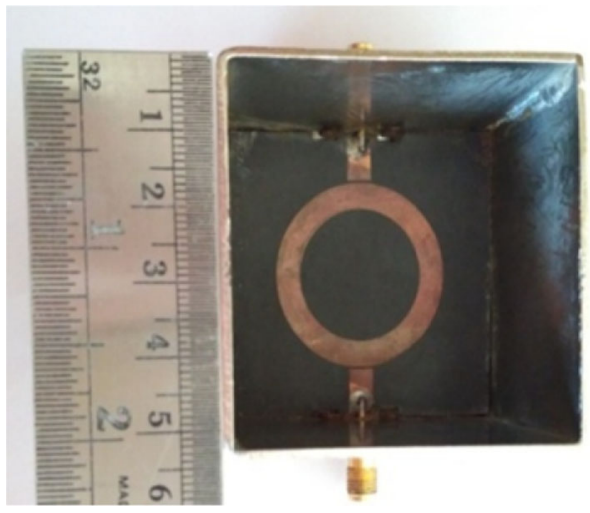

Figure 2. Open boxed resonator.

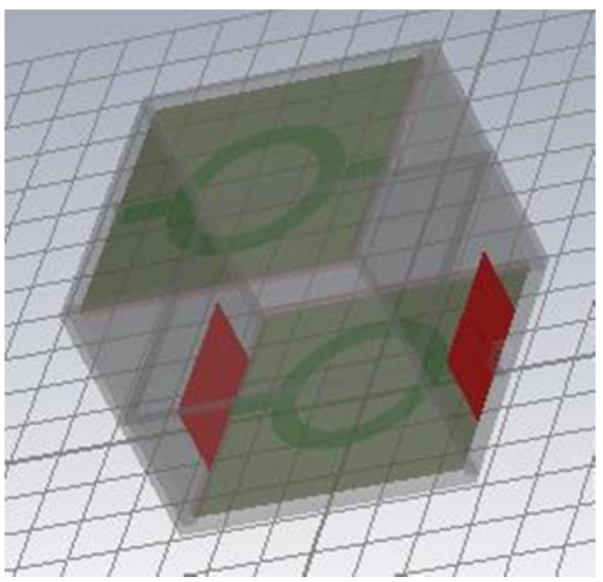

Figure 3. Stacked ring resonator $3 \mathrm{D}$ model.

ring resonator structure $[13,14]$. The microstrip ring resonator structure is a closed loop transmission line structure. Resonance occurs when the mean circumference of the ring is equal to an integral multiple of guided wavelength as given in Eq. (1). In Eq. (1) ' $r$ ' denotes mean radius of microstrip ring and $\lambda$ is wavelength. Microstrip ring resonator is made with RT Duroid 5880 substrate having thickness $1.575 \mathrm{~mm}$. In this case, assuming $\mathrm{n}=1$ and resonating frequency $2.45 \mathrm{GHz}$ calculated $\lambda$ is $89.2 \mathrm{~mm}$. Figure 1 shows planar ring resonator structure.

$$
2 \pi \mathrm{r}=\mathrm{n} \lambda
$$

Table 1 describes width to height $\mathrm{w} / \mathrm{h}$ ratio, Effective Permittivity $\varepsilon_{\text {eff }}$, Impedance $\mathrm{Z}_{\mathrm{o}}$ of the planar resonator of RT Duroid and FR4 substrate. Table 2 describes $w$ and $r$ for various substrates used in the simulation for selection of substrates for fed patch and parasitic patch. Simulation results show best results with RT Duroid 5880 material having $1.575 \mathrm{~mm}$ thickness. It is selected for the fed patch. CST MWS was utilized for fine tuning the 3D model of 
Table 1. Calculations of microstrip ring impedance.

\begin{tabular}{lccccc}
\hline $\begin{array}{l}\text { Relative } \\
\text { permittivity } \varepsilon_{\mathrm{r}}\end{array}$ & $\begin{array}{c}\text { Width } \\
\mathrm{W}(\mathrm{mm})\end{array}$ & $\begin{array}{c}\text { Height } \\
\mathrm{h}(\mathrm{mm})\end{array}$ & $\mathrm{w} / \mathrm{h}$ & $\begin{array}{c}\text { Effective } \\
\text { Permittivity } \varepsilon_{\mathrm{eff}}\end{array}$ & $\begin{array}{c}\text { Impedance } \mathrm{Z}_{\mathrm{O}} \\
(\text { Ohms })\end{array}$ \\
\hline 2.2 & 4.8 & 1.575 & 3.05 & 1.87 & 50.66 \\
4.3 & 3.087 & 1.6 & 1.93 & 3.26 & 50.49 \\
\hline
\end{tabular}

Table 2. Different substrate used with different $w / r$ ratios.

\begin{tabular}{|c|c|c|c|c|c|}
\hline Substrate & Dielectric constant & Loss tangent & Thickness (mm) & $\begin{array}{l}\text { Mean radius } \\
\text { 'r' }(\mathrm{mm}) \text { of } \mathrm{Cu}\end{array}$ & $\begin{array}{c}\text { Width } \\
\text { 'w' (mm) of } \mathrm{Cu}\end{array}$ \\
\hline RT Duroid & 2.2 & 0.0009 & 0.762 & 14.200 & 2.36 \\
\hline RT Duroid & 2.2 & 0.0009 & 1.575 & 14.200 & 4.80 \\
\hline GML 1000 & 3.2 & 0.0004 & 0.762 & 12.200 & 1.84 \\
\hline FR4 Epoxy & 4.4 & 0.0250 & 1.600 & 10.690 & 3.00 \\
\hline Alumina & 9.8 & 0.0001 & 0.508 & 7.585 & 0.49 \\
\hline
\end{tabular}

boxed ring resonator designed to achieve a high Q-factor with the following considerations. $Z_{\mathrm{o}}$ of the transmission line was kept $50 \Omega$ for impedance matching of the source and feed-line. Coupling gap was adjusted and optimised to $0.3 \mathrm{~mm}$ to reduce the insertion loss but also to maintain Quality factor. $Z_{0}$ and $\varepsilon_{\text {eff }}$ were less affected as the substrate width was kept 10 times greater than the width of the copper strip. The thickness of the conductor box was selected to $2 \mathrm{~mm}$. The thickness has been kept greater than five times the skin depth of the conductor. Similarly, the height of box was optimized for higher $\mathrm{Q}$ and lower sample volume.

Figure 2 shows open boxed structure without top cover. A steel sheet with $2 \mathrm{~mm}$ thickness is used for shielding. Box height is kept half wavelength i.e., $44.75 \mathrm{~mm}$ from the surface of the substrate. Stacked Ring Resonator is formed after covering the open box with planar microstrip structure. Figure 3 shows simulated 3D stacked structure.

\section{Stacked multi ring resonator}

Design and experimentation are carried out with planar resonator, the boxed ring resonator, stacked ring resonator and multiring resonator to improve quality factor $\mathrm{Q}$ with minimum return loss. During experimentation of the stacked resonator, there is a small second peak in frequency response due to the mismatch in the fed patch and parasitic patch peak frequency. This is one of the shortcomings of stacked structure [5]. Multiring stacked structure helps to remove the second peak. The following section describes selection and design of multiring resonator.

\subsection{Working principle of stacked resonator}

As the fringing field for low permittivity substrate is more, we have selected RT Duroid substrates as the fed patch to measure the dielectric constant. Two resonances are associated due to the presence of the parasitic element in the stacked microstrip ring resonator. First resonance is associated with the lower patch i.e., fed patch and the ground plane. The second resonance is associated with the upper patch i.e., the parasitic patch and lower patch. The first resonance is considered as a microstrip patch with the material sample (superstrate). Due to the material under test $M U T$, the effective dielectric constant $\varepsilon_{\text {eff }}$ is changed, and the resonance frequency will decrease with increase in sample thickness and the dielectric constant of the superstrate [11].

\subsection{Selection of substrate for parasitic patch}

Experimentation is done for the boxed resonator with and without metal cover. The Quality factor of 125 at the bandwidth of $20 \mathrm{MHz}$ is measured for boxed resonator without cover. For further experimentation, the box is covered with the inverted parasitic patch. Parasitic patch ground is top surface of SMRR while rings on parasitic patch are facing inside the box. A gap $h p$ of $0.45 \lambda$ $(0.45 * 89.2 \mathrm{~mm}=40.14 \mathrm{~mm}) \quad$ and $\quad 0.50 \lambda$ $(0.50 * 89.2 \mathrm{~mm}=44.75 \mathrm{~mm})$ is kept between the fed patch and parasitic patch. It is observed that input impedance $\mathrm{Z}_{11}$ is maximum when $h p$ is $0.45 \lambda$. The quality factor is measured by changing top cover with different substrates like alumina $\left(\varepsilon_{\mathrm{r}}=9.8\right)$, FR4 Epoxy $\left(\varepsilon_{\mathrm{r}}=4.4\right)$, GML $1000\left(\varepsilon_{\mathrm{r}}=3.2\right)$ and the different number of rings etched on it [15]. Figure 4 demonstrates the effect of $Q$ due to different substrates used for the parasitic patch. Nelco GML1000 substrate patch with four rings is giving highest $\mathrm{Q}$ with two resonant frequencies in simulation results. But the desired condition is to have the single resonant frequency, so the FR4 substrate is selected for further investigation rather than GML1000. So FR4 substrate parasitic patches are made with 2, 3 and 4 rings. 


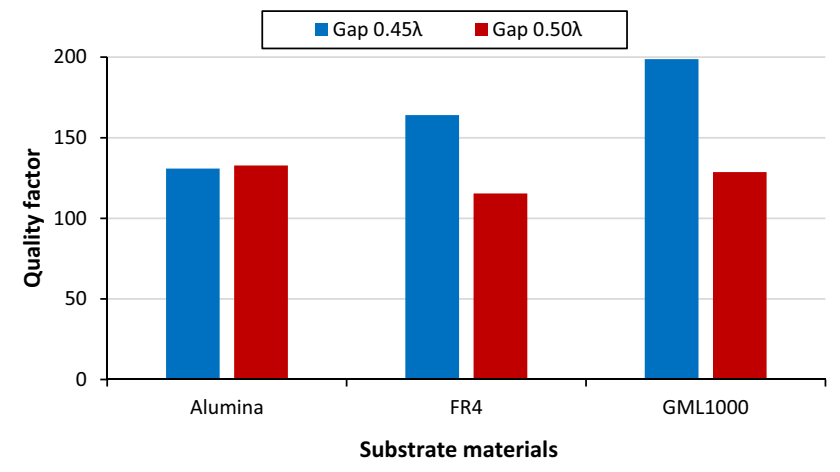

Figure 4. Effect on Q due to different parasitic patch substrates.

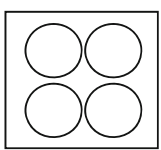

(a)

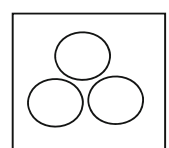

(b)

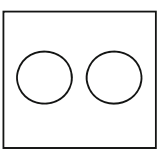

(c)

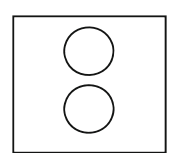

(d)
Figure 5. (a) 4 rings, (b) 3 rings, (c) 2 rings even mode and (d) 2 rings odd modes.

\subsection{Multiple rings parasitic patch}

The parasitic patch is developed with the FR4 substrate. Four, three and two rings are etched on the parasitic patch

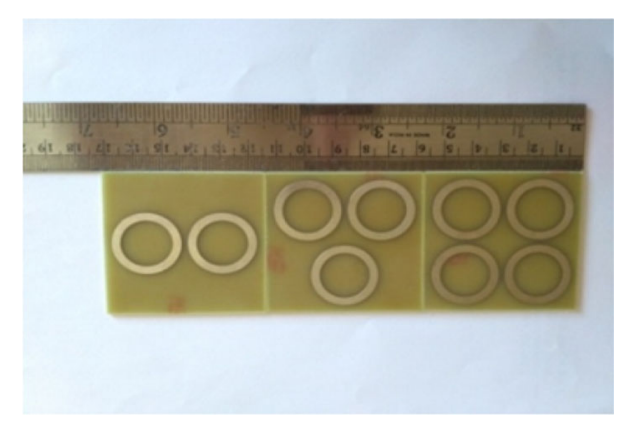

Figure 6. Parasitic patches with FR 4 substrate.

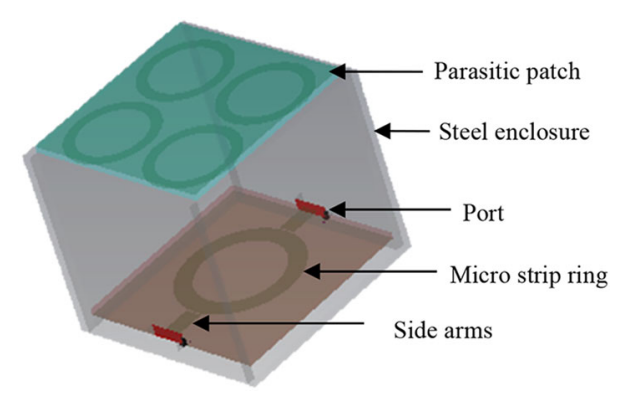

Figure 7. SMRR with 4 rings on parasitic patch and single ring on fedpatch. as per figure $5(\mathrm{a})-(\mathrm{d})$. The width and mean radius of rings on FR4 is $3 \mathrm{~mm}$ and $10.69 \mathrm{~mm}$, respectively. Figure 6 shows fabricated FR4 substrates with multiple rings. Figure 7 shows stacked resonator with steel enclosure. SMRR has four rings on the parasitic patch and single ring on the fed patch.

4 Rings: placed at equal distance from center

3 Rings: placed 2 rings at equal distance and one ring at center axis.

2 Rings Even mode: 0 degree with fed ring patch

2 Rings Odd mode: 90 degree with fed ring patch

Experimentation is carried out with 2 rings even mode i.e., 0 degrees with fed ring patch and 2 rings odd mode i.e., 90 degrees with fed ring patch, 3 rings and 4 rings parasitic patches [16]. Electric field $E$ distribution increases above $0.3 \lambda$ and decreases after $0.7 \lambda$ in SMRR. The electromagnetic field is maximum at $0.45 \lambda$. Table 3 shows the simulated result of $\mathrm{E}$ field, $\mathrm{H}$ field and surface current with FR4 patch kept at the $0.45 \lambda$ distance from the fed patch. As per table 3 maximum $Q$, narrower bandwidth, minimum loss $S_{21}$ and maximum $E$ field coupling are obtained with 4 rings SMRR. Finally, 4 rings FR4 patch is selected for dielectric measurement of low permittivity material between 1 and 4 .

\subsection{Sample height effect in stacked resonator}

As overlay thickness increases resonating frequency decreases. As more fringing field is getting concentrated into the sample, it increases fringing field capacitance and decreases resonating frequency. Beyond $0.04 \lambda$ thickness, its effect is not significant. Effective permittivity increases with overlay height $(0.01 \lambda$ to $0.03 \lambda)$ until it reaches asymptotic value, it indicates all electric field is confined to overlay and substrate [3]. Change in $f r$ was seen within the range of $0.8 \%$ to $1 \%$ of the desired $f r$ for SMRR and moderate $\mathrm{Q}=160$. The $\mathrm{Z}_{11}$ magnitude impedance shows negligible changes for thin overlays but decreases for thicker overlays [4]. We have considered the sample height $0.02 \lambda$ or $5 \mathrm{ml}$ volume for experimentation.

\subsection{Analysis of losses}

The microstrip loss analysis and stacked structure are fairly complicated due to the presence of the dielectric-air interface. Analysis of loss calculation is based on assumption that electric and magnetic field distribution in the structure is not altered due to the presence of losses. Simple equivalent circuit and electromagnetic theory for ring resonator are discussed by Hopkins et al [17]. The dielectric constant can be extracted by measuring resonant frequency and quality factor of the circuit [17]. There are three types of losses that occur in microstrip lines and they include the conductor loss, the dielectric loss and the radiation loss. 
The conductor loss and the dielectric loss are the main losses in microstrip line devices, while the radiation loss normally neglected. Losses are simulated with the planar, boxed, stacked and SMRR structure using CST MWS software.

$\mathrm{Q}$ is the measure of power loss in the resonant circuit. As higher $\mathrm{Q}$ is desirable in dielectric characterization applications that mean lower losses in a resonator is needed. Manufacturing loss is around $1.3 \mathrm{~dB}$ for the resonator. Losses in the planar resonator are maximum around $9 \mathrm{~dB}$. When the planar resonator is enclosed with the metal enclosure, return losses are reduced by $3 \mathrm{~dB}$. As coupled microstrip lines are enclosed the Electric Field E in air terminates prematurely at walls of the box which gives rise to an increase in the mode of capacitance. Therefore, Q increases and impedance decreases. If boxed ring resonator is covered with metal sheet losses are reduced by $1 \mathrm{~dB}$ than open box readings. Losses are reduced due to dual resonance in the stacked structure by around $4 \mathrm{~dB}$ than planar structure. Both top cover and side wall tends to lower impedance and therefore effective dielectric constant $\varepsilon_{\text {eff }}$ reduces. The effective dielectric constant of planar ring resonator is 1.87 which is reduced to 1.67 for stacked ring resonator.

For a gap coupled ring resonator, the coupling region has the maximum electric field but more sensitive to overlay permittivity variations. Gap capacitance deviates inherent resonant frequency to lower frequency which is known as pushing effect [18]. This pushing effect lowers insertion loss, but the effect on resonant frequency is significant. Typically, S21 is used to determine insertion loss and reduces by increasing metallic shielding in boxed structure. In SMRR cavity is completely shielded, there is no radiation effect. Shielded box or metal housing plays an important role in the performance of SMRR. Experimental results show that losses are reduced in SMRR structure by $50 \%$ than planar resonator as per Table 4. The loss is minimum with SMRR structure.

Theoretical estimation of conductor loss $\alpha_{\mathrm{C}}$ in RT Duroid 5880 for $2.45 \mathrm{GHz}$ is $0.0483 \mathrm{~Np} / \mathrm{m}$ or $0.4192 \mathrm{~dB} / \mathrm{m}$ considering surface resistivity $0.00128 \mathrm{Ohms}$, and skin depth is 1.33 micrometre. Here copper ring is having width ' $\mathrm{w}$ ' is $4.8 \mathrm{~mm}$ and thickness ' $\mathrm{t}$ ' of copper 17.5 micrometre on RT Duroid substrate. Theoretical dielectric loss $\alpha_{d}$ is 0.02693 $\mathrm{Np} / \mathrm{m}$ or $0.2339 \mathrm{~dB} / \mathrm{m}$. Losses are also verified by 'Roggers
Table 4. Comparison of experimental and simulated measurement.

\begin{tabular}{lccccc}
\hline & \multicolumn{2}{c}{ Simulated } & & \multicolumn{2}{c}{ Experimental } \\
\cline { 2 - 3 } \cline { 5 - 6 } $\begin{array}{l}\text { Resonator } \\
\text { structures }\end{array}$ & $\begin{array}{c}\mathrm{S}_{21} \text { in } \\
\mathrm{dB}\end{array}$ & $\begin{array}{c}\text { Loaded } \\
\mathrm{Q}_{\mathrm{L}}\end{array}$ & & $\begin{array}{c}\mathrm{S}_{21} \text { in } \\
\mathrm{dB}\end{array}$ & $\begin{array}{c}\text { Loaded } \\
\mathrm{Q}_{\mathrm{L}}\end{array}$ \\
\hline Planar & -7.49 & 73 & & -8.76 & 69 \\
Boxed & -4.31 & 125 & & -6.36 & 119.19 \\
Metal covered box & -3.65 & 137.79 & & -5.69 & 133.57 \\
Stacked & -2.91 & 154.74 & & -4.98 & 146.67 \\
SMRR & -2.61 & 160 & & -4.05 & 154.4 \\
\hline
\end{tabular}

Impedance Calculator' for planar structure. The analysis of losses in microstrip ring structure is becoming more and more important for developed SMRR structure. Table 5 describes simulated quality factor, total attenuation $\alpha_{\mathrm{T}}$ and phase constant $\beta$ for various structures. Theoretical and simulated unloaded quality factor comparison is made, and it showed good agreement.

Typically, insertion loss $S_{21}$ is used to determine insertion loss and reduces by increasing metallic shielding with the planar structure. $Q$ unloaded values can be calculated by Eq. (2) from simulated or experimental values of $Q$ loaded and insertion loss $S_{21}$. Loaded $Q_{L}$ and unloaded quality factors $\mathrm{Q}_{\mathrm{u}}$, the relationship is as per Eq. (2) [19]. Equation (3) says that total attenuation is the summation of conductor and dielectric attenuations. Unloaded quality factor $\mathrm{Q}_{\mathrm{u}}$ of microstrip is related to total attenuation $\alpha_{\mathrm{T}}$ and $\beta$ in microstrip as per Eq. (4) [20]. The simulated data are summarized and cross checked with theoretical values. It is observed that attenuation $\alpha$ is higher in planar ring structure which is five times larger than SMRR structure. No significant change in beta is observed. Table 5 data depict that SMRR sensor is more accurate than simple planar structure.

$$
\begin{gathered}
Q_{u}=\frac{Q_{L}}{1-10^{-L / 20}} \\
\alpha_{T}=\alpha_{C}+\alpha_{d} \\
Q_{u}=\frac{\beta}{2 \alpha_{T}}
\end{gathered}
$$

\begin{tabular}{|c|c|c|c|c|}
\hline Parasitic patch & 4 rings & 2 rings even & 2 rings odd & 3 rings \\
\hline Resonating frequency $f r(\mathrm{GHz})$ & 2.463 & 2.458 & 2.458 & 2.459 \\
\hline $\mathrm{S}_{21}(\mathrm{~dB})$ & -2.61 & -3.637 & -3.83 & -2.95 \\
\hline Bandwidth (MHz) & 15.26 & 32.3 & 25 & 19 \\
\hline Q & 160.15 & 76.64 & 97.92 & 129.42 \\
\hline E field V/m & $341.4 \mathrm{e}+3$ & $153.8 \mathrm{e}+3$ & $216.1 \mathrm{e}+3$ & $231.2 \mathrm{e}+3$ \\
\hline H Field A/m & 674.2 & 297 & 920 & 525.6 \\
\hline Surface current A & 682.9 & 312.4 & 918 & 526 \\
\hline
\end{tabular}

Table 3. Various parameters with 2, 3 and 4 rings of SMRR. 
Table 5. Theoretical and simulated unloaded quality factor Q.

\begin{tabular}{|c|c|c|c|c|c|c|}
\hline Resonators & $\begin{array}{c}\mathrm{S}_{21} \text { in } \\
\mathrm{dB}\end{array}$ & $\begin{array}{c}\mathrm{Q}_{\mathrm{L}} \\
\text { Loaded }\end{array}$ & $\mathrm{Q}_{\mathrm{u}}$ Simulated & $\begin{array}{c}\alpha_{\mathrm{T}} \\
\mathrm{Np} / \mathrm{m}\end{array}$ & $\beta \mathrm{rad} / \mathrm{m}$ & $\begin{array}{c}\mathrm{Q}_{\mathrm{u}} \\
\text { Theoretical }\end{array}$ \\
\hline Planar & -7.49 & 73 & 126.337 & 0.2625 & 66.47 & 126.602 \\
\hline Open boxed & -4.31 & 125 & 319.559 & 0.1042 & 66.66 & 319.925 \\
\hline Stacked & -2.91 & 154.74 & 543.085 & 0.0611 & 66.42 & 543.745 \\
\hline SMRR (4 rings) & -2.61 & 160 & 616.47 & 0.0539 & 66.4 & 616.527 \\
\hline
\end{tabular}

where $\alpha_{\mathrm{T}}$ Total attenuation $\mathrm{Np} / \mathrm{m}, \alpha_{\mathrm{c}}$ Attenuation due to conductor loss Np/m, $\alpha_{\mathrm{d}}$ Attenuation due to dielectric loss $\mathrm{Np} / \mathrm{m}, \mathrm{Q}_{\mathrm{u}}$ Unloaded quality factor, $\mathrm{Q}_{\mathrm{L}}$ Loaded quality factor.

\subsection{Complex permittivity measurement}

The novel SMRR structure is used for low loss liquid complex permittivity measurement.

Different methods have been reported which give accurate results of relative permittivity at microwave frequencies. The real part of the permittivity $\varepsilon_{\mathrm{r}}^{\prime}$ also called the dielectric constant, affects the electric field and wave impedance of a propagating wave, whereas the imaginary part of the permittivity $\varepsilon_{\mathrm{r}}^{\prime \prime}$ indicates how lossy a medium is. Real and imaginary part of the dielectric constant of a homogeneous medium in contact with ring resonator is calculated from the shift in the resonant frequency and change in the quality factor of the resonator [21]. Complex permittivity can be given as per Eq. (5)

$$
\varepsilon_{\mathrm{r}}=\varepsilon_{\mathrm{r}}^{\prime}-\mathrm{j} \varepsilon_{\mathrm{r}}^{\prime \prime}
$$

SMRR is multilayer structure [22] which contains dielectric substrate, the material under test and air. Effective permittivity is calculated under unloaded condition [6]. When the test sample is loaded in the resonator, there is change in the effective permittivity. The real part of dielectric constant for test sample $\left(\varepsilon_{\text {mut }}\right)$ is calculated using the practically obtained value of the $\varepsilon_{\text {eff }}$.

The resonant frequencies of the loaded and unloaded resonator are $\mathrm{f}_{1}$ and $\mathrm{f}_{\mathrm{ul}}$, respectively. The resonant frequency is measured from the spectrum of $\mathrm{S}_{21}$ with VNA. Using Eq. (6) the real part of the effective dielectric constant of the loaded resonator $\varepsilon_{\text {effl }}$ and unloaded resonator $\varepsilon_{\text {efful }}$ is calculated in terms of measured $\mathrm{f}_{1}$ and $\mathrm{f}_{\mathrm{ul}}$ [11].

$$
\frac{f_{u l}^{2}}{f_{l}^{2}}=\frac{\epsilon_{e f f l}}{\epsilon_{e f f u l}}
$$

Kobayashi [23], Kirschning et al [24] depicted that dielectric constant can be deduced from effective permittivity $\varepsilon_{\text {eff }}$ for microstrip resonator. To predict complex permittivity of test sample, effective permittivity should be calculated. Rueggeberg [18] proposed complex permittivity measurement method for arbitrarily dimensioned dielectric modules at microwave frequencies. 3.6a Calculation of real part of permittivity $\varepsilon_{r}$ ': Relative permittivity can be calculated from the shift in resonant frequency. SMRR 3D model simulations were carried out for unloaded and loaded conditions for material having permittivity 1 to 7 for the $5 \mathrm{ml}$ of volume. Rueggeberg [18] proposed that the dimensionless ratio $\left(\mathrm{f}_{0} / \mathrm{f}_{\text {mut }}\right)$ may be advantageously used for prediction of the real part of permittivity where $f_{o}$ is unloaded frequency and $f_{\text {mut }}$ is loaded frequency. Using CST MWS 2016, frequency response data is plotted and using polynomial curve fitting permittivity is predicted. The real part of permittivity can be calculated as per polynomial Eq. (7) where $\mathrm{x}=\mathrm{f}_{\mathrm{o}} / \mathrm{f}_{\text {mut }}$ and $\mathrm{y}=$ relative permittivity.

$$
y=-249.8 x^{3}+884.3 x^{2}-1015 . x+382.0
$$

After the desired resonance curve has been obtained, the evaluation of an unknown sample permittivity $\varepsilon_{\mathrm{r}}$ is simply the measurement of the resonant frequency. Figure 8 shows resonance frequency characteristics curve for $\left(\mathrm{f}_{\mathrm{o}} / \mathrm{f}_{\text {mut }}\right)$ with respect to relative permittivity $\varepsilon_{\mathrm{r}}$ The dielectric constant is evaluated directly from the sensor resonant frequency characteristic as per Eq. (7).

3.6b Calculation of imaginary part of permittivity $\varepsilon_{r}^{\prime \prime}$ : When the finitely thick layer of liquid is placed above the microstrip ring resonator, the energy is dissipated in three different regions. The effective permittivity can be expressed as a linear combination of the permittivity of the air $\left(\varepsilon_{\mathrm{r}}=1\right)$, the substrate $\left(\varepsilon_{\mathrm{sub}}\right)$ and the material under test $\left(\varepsilon_{\text {mut }}\right)$. To measure the imaginary part of the permittivity, determination of dissipation factor $\tan \delta$ is essential.

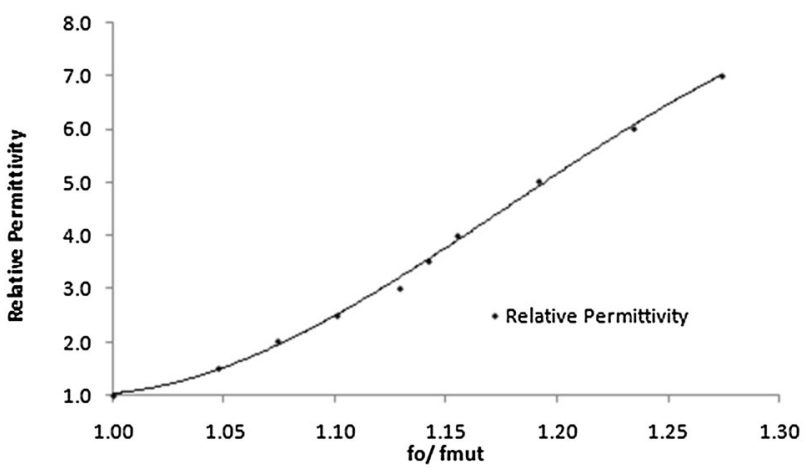

Figure 8. Resonance frequency characteristics curve for $f_{o} / f_{\text {mut }}$ of relative permittivity. 


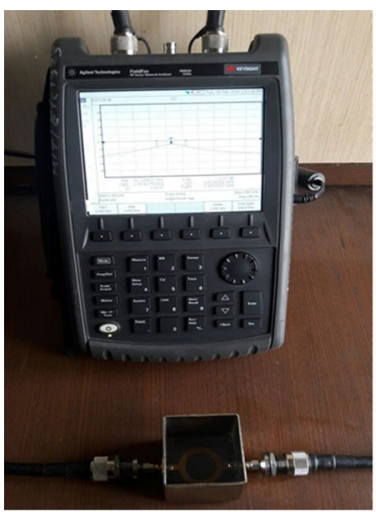

(a)

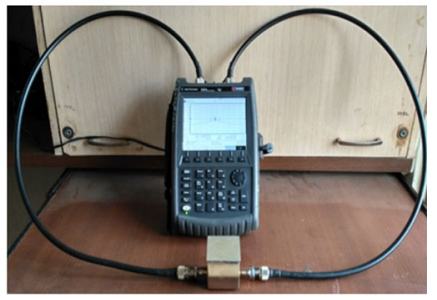

(b)
Figure 9. (a) Experimental set-up for boxed resonator without parasitic patch. (b) Experimental set-up for SMRR.

Determination of the values of dissipation factor is based on measuring the loaded quality factor $Q_{L}$ and $S_{21}$ at each resonant frequency. The unloaded quality factor $\mathrm{Q}_{\mathrm{u}}$ which includes total losses, can be calculated from the measured value of $\mathrm{Q}_{\mathrm{L}}$. With careful fabrication procedure, the input and output air gap can be made equal to each other. Thus coupling coefficient $\mathrm{k} 1$ from input side and $\mathrm{k} 2$ from output side proves equation $\mathrm{k} 1=\mathrm{k} 2$. For such symmetrical coupling, $\mathrm{Q}_{\mathrm{u}}$ can be obtained as per Eq. (8) [5, 15].

$$
Q_{u}=\frac{Q_{L}}{1-s_{21}\left(f_{0}\right)}
$$

Loss tangent tan $\partial$ of the dielectric material is a quantity used to describe the dielectric properties that influences reflection of the electromagnetic wave at interfaces and attenuation of wave energy within materials [21]. The loss tangent is given by following Eqs. (9), (10), (11), (12) [25-27].

$$
\begin{gathered}
\varepsilon_{r}^{\prime \prime}=\varepsilon_{r}^{\prime}(1-\mathrm{j} \tan \partial) \\
\tan \delta=\frac{\varepsilon_{r}^{\prime \prime}}{\varepsilon_{r}^{\prime}} \\
\frac{1}{Q_{u}}=\frac{1}{Q_{c}}+\frac{1}{Q_{d}}+\frac{1}{Q_{r}} .
\end{gathered}
$$

$$
\tan \delta=\frac{1}{Q_{u}}
$$

Equation (11) represents total quality factor $\mathrm{Qu}$ for the multilayer SMRR. Qc, Qd, Qr represent Q-factors due to conductor loss, dielectric loss and radiation loss. Radiation loss is negligible for shielded SMRR compared to dielectric and conductor loss of the SMRR. Major contribution in loss factor is conductor and dielectric loss. For low loss, material loss tangent can be calculated as per Eq. (12).

\subsection{Experimental set-up of dielectric constant measurement}

Experimentation is done with Agilent Technologies RF Vector Network Analyser N9923A at room temperature and assuming humidity $50 \%$. Parameters like $\mathrm{S}_{21}$, resonating frequency, bandwidth and $Q_{L}$ have been measured as per measurement set-up shown in figure 9(a) and (b). Measurement is done using 801 data points and $1 \mathrm{kHz}$ resolution $\mathrm{BW}$ gives a maximum resolution of $100 \mathrm{~Hz}$ in the measurement range of the frequency 2 to $2.6 \mathrm{GHz}$. All testing is done in two port transmission modes. Frequency measurement was kept for all reading from 2 to $2.6 \mathrm{GHz}$ as the designed structure is for $2.45 \mathrm{GHz}$. MUT is filled in the SMRR sensor up to $0.02 \lambda$ height or $5 \mathrm{ml}$ in volume. Resonating frequency is very sensitive to the dielectric constant of MUT.

Different liquid or paste test samples with permittivity in the range of 1 to 4 like $\mathrm{N}$ hexane, kerosene, diesel, wax, lubricant oil and crude oil are tested. N-hexane and wax are purchased from Sharad Chemicals, Pune. Diesel and crude oil purchased from Shell Petrol Pump, Pune. Kerosene and lubricant oil is purchased from standard outlets. Various parameters like resonating frequency, $\mathrm{S}_{21}$, bandwidth, $\mathrm{Q}_{\mathrm{L}}$ are measured using VNA. Real part of permittivity $\varepsilon_{\mathrm{r}}{ }^{\prime}$ is calculated using resonant frequency reading and Eq. (7). Loss tangent is calculated using Eqs. (8) and (12). Imaginary part of permittivity $\varepsilon_{\mathrm{r}}^{\prime \prime}$ is calculated as per Eq. (10).

\begin{tabular}{|c|c|c|c|c|c|c|c|}
\hline Material & $\begin{array}{l}\text { Mean resonant frequency } \\
(\mathrm{MHz})\end{array}$ & $\begin{array}{l}\text { Mean quality factor } \\
\left(\mathrm{Q}_{\mathrm{L}}\right)\end{array}$ & $\begin{array}{l}\text { Mean } S_{21} \\
\quad(d B)\end{array}$ & $\begin{array}{c}\mathrm{BW} \\
(\mathrm{MHz})\end{array}$ & $\begin{array}{c}\text { Mean } \\
\varepsilon_{\mathrm{r}}^{\prime}\end{array}$ & $\begin{array}{c}\text { Tan } \\
\text { delta }\end{array}$ & $\varepsilon_{\mathrm{r}}^{\prime \prime}$ \\
\hline $\begin{array}{c}\text { Without } \\
\text { sample }\end{array}$ & 2442 & 154.4 & -4.05 & 15.81 & - & - & - \\
\hline N-hexane & 2343 & 99.87 & -6.65 & 23.46 & 1.895 & 0.00536 & 0.01015 \\
\hline Wax & 2336 & 110.19 & -7.36 & 21.20 & 1.939 & 0.00519 & 0.01006 \\
\hline Diesel & 2325 & 78.32 & -7.71 & 29.69 & 2.016 & 0.00884 & 0.01782 \\
\hline Kerosene & 2314 & 77.1 & -8.88 & 30.01 & 2.097 & 0.00830 & 0.01741 \\
\hline Crude oil & 2303 & 77.82 & -6.91 & 29.59 & 2.183 & 0.00705 & 0.01539 \\
\hline Lubricant oil & 2299 & 68.61 & -8.61 & 33.51 & 2.219 & 0.00917 & 0.02034 \\
\hline
\end{tabular}
Therefore, complex permittivity can be determined by Eq. (5). Table 6 shows complex permittivity calculations for different MUTs from experimental readings.

Table 6. Experimental readings for complex permittivity measurement. 


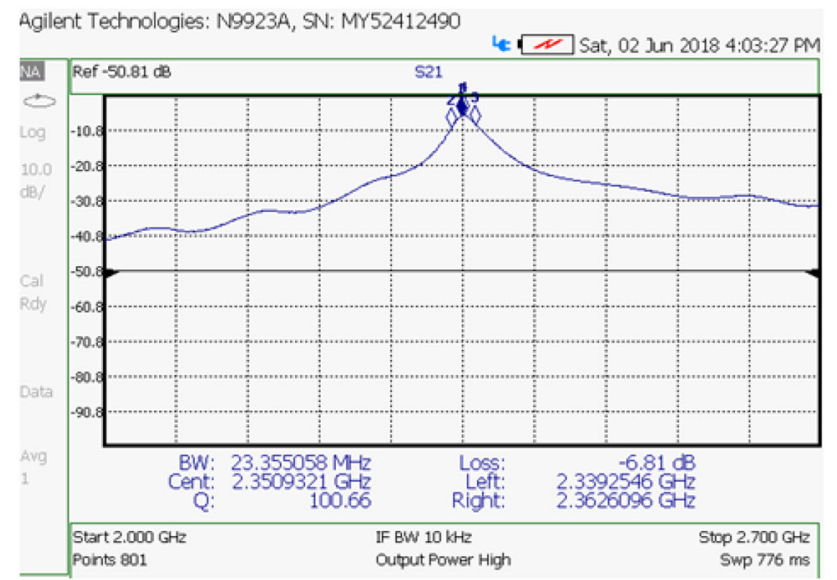

Figure 10. Measured return loss and frequency of SMRR with N-hexane.

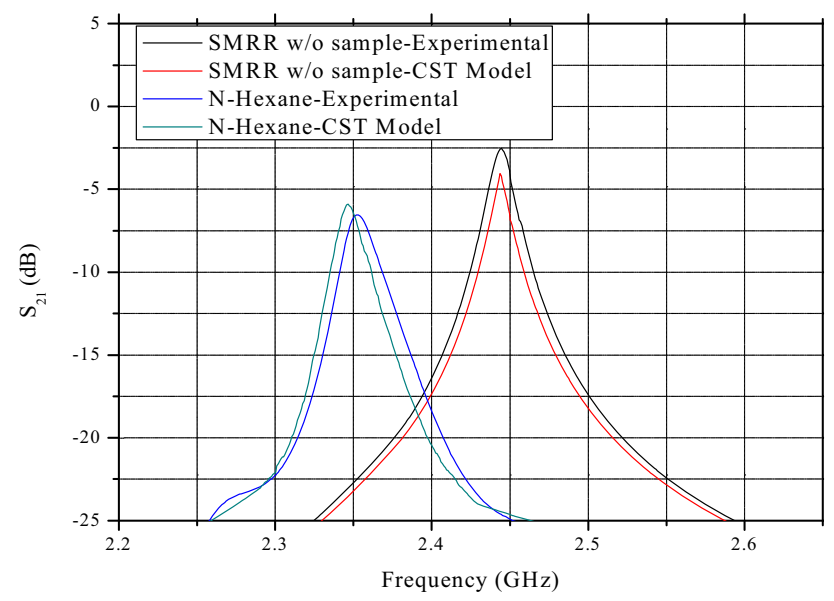

Figure 11. Comparison of experimental and simulated frequency response of SMRR with and without test sample.

Figure 10 shows variation in $S_{21}$ against frequency plot of N-Hexane sample from Agilent Technologies VNA N9923. Figure 11 provides the comparison between experimental and CST MWS simulated $\mathrm{S}_{21}$ parameters for SMRR without test sample and SMRR with test sample as N-Hexane.

Sensitivity is evaluated as change in resonating frequency with respect to change in relative permittivity [26]. Sensitivity reported for the stacked structure is $13.73 \mathrm{MHz}$ per $1 \%$ change in relative permittivity [5]. Sensitivity with developed SMRR structure is $15.02 \mathrm{MHz}$ per $1 \%$ change in relative permittivity. It is observed that attenuation alpha can be calculated synthetically by using experimental data of resonating frequency, insertion loss and loaded quality factor. Line impedance varies between 31 and $33 \mathrm{Ohms.}$ Copper loss reduces as permittivity increases. Developed 3D SMRR model in CST MWS shows dielectric constant results in good agreement with experimental data as shown in figure 11. This analysis confirms the suitability of the structure for low loss liquid permittivity measurement.

Dielectric constant is verified on Keysight Vector Network Analyzer PNA E834C with 85070E dielectric probe kit. It contains coaxial probe system. It uses reflection coefficient system to calculate dielectric constant. We have measured dielectric constant and loss tangent of water and then test samples of liquids and paste.

\section{Discussion of quality factor enhancement with SMRR}

The stacked structure contains parasitic patch (upper inverted ring resonator) and the fed patch (lower ring resonator). We have mainly analysed electromagnetic field and impedance bandwidth for SMRR structure with CST MWS to study the effect of coupling and enhancement in quality factor with number of rings.

\subsection{Electromagnetic field analysis of SMRR}

The study shows that $\lambda / 2$ space between the fed patch and the parasitic patch forms the resonator. In near-field analysis by the TLM method makes it clear that electromagnetic field is coupled. So, when the electromagnetic field is coupled the magnitude of electric fields in the cavity becomes large. The magnitude of current distribution on the parasitic patch also becomes large. Table 7 describes the synthetic analysis of $\mathrm{E}$ and $\mathrm{H}$ field quantities. $\mathrm{E}$ field and $\mathrm{H}$ field for stacked ring resonator are as shown in figures 12 and 13, respectively. E field and $\mathrm{H}$ field for SMRR are as shown in figures 14 and 15, respectively. EM field coupling is more in stacked resonator than top metal cover boxed resonator as shown in table 7 . It is observed that $E$ field in SMRR is maximum. The surface current due to metal sheet cover is maximum in boxed with the covered metal sheet. $\mathrm{H}$ field coupling in the boxed resonator with metal cover is maximum. Due to parallel resonance between the lower fed patch and upper parasitic patch capacitive coupling increases and plays an important role in increasing $E$ field. In addition to that as number of rings in parasitic patch increases, electric field enhances. As per table $3 \mathrm{E}$ field increases as number of rings increases. Electromagnetic field coupling is maximum for four rings. Equation (13)

Table 7. Comparison of $\mathrm{E}$ and $\mathrm{H}$ field for different resonators.

\begin{tabular}{lcc}
\hline Resonators & E field V/m & H field A/m \\
\hline Planar resonator & $148.4 \mathrm{e}+03$ & 127.2 \\
Open boxed resonator & $207.1 \mathrm{e}+03$ & 188.3 \\
Boxed with metal cover resonator & $222.8 \mathrm{e}+03$ & 274 \\
Stacked resonator & $225.5 \mathrm{e}+03$ & 230 \\
SMRR (4 rings) & $309.3 \mathrm{e}+03$ & 249.4
\end{tabular}




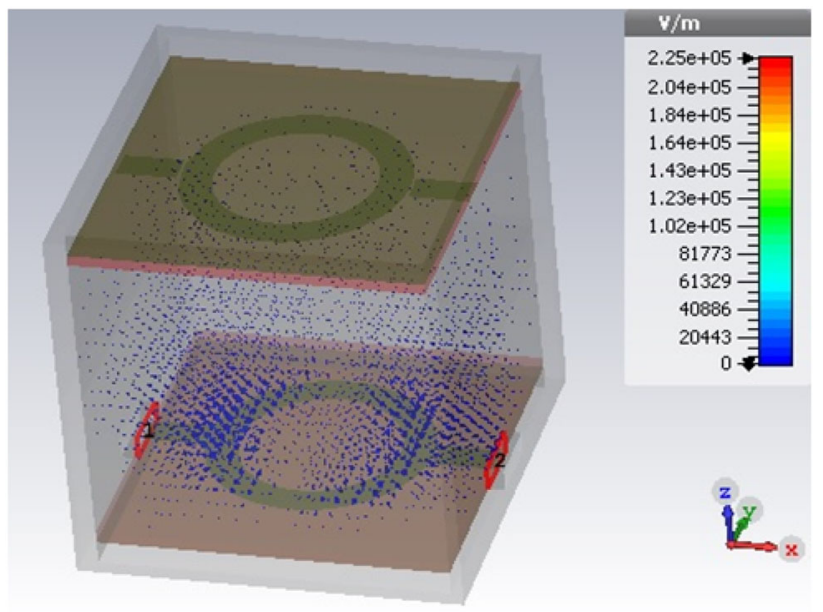

Figure 12. E Field- stacked resonator.

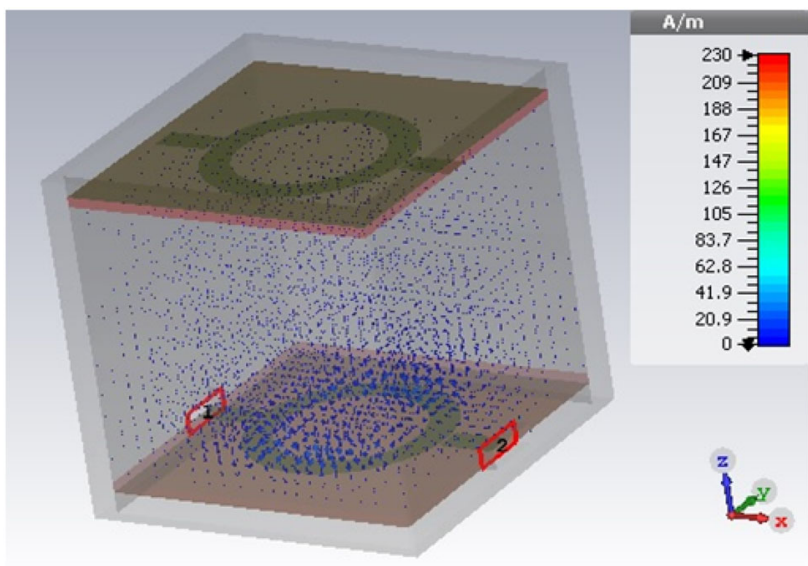

Figure 13. H Field- stacked resonator.

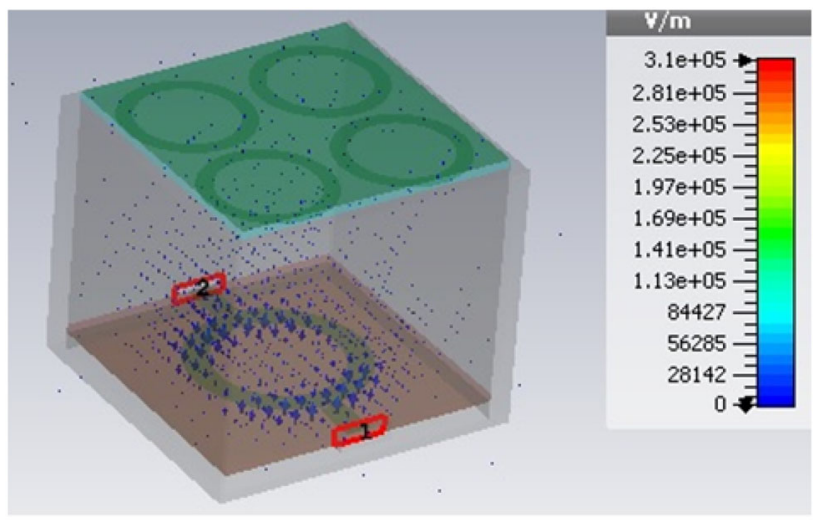

Figure 14. E Field for SMRR.

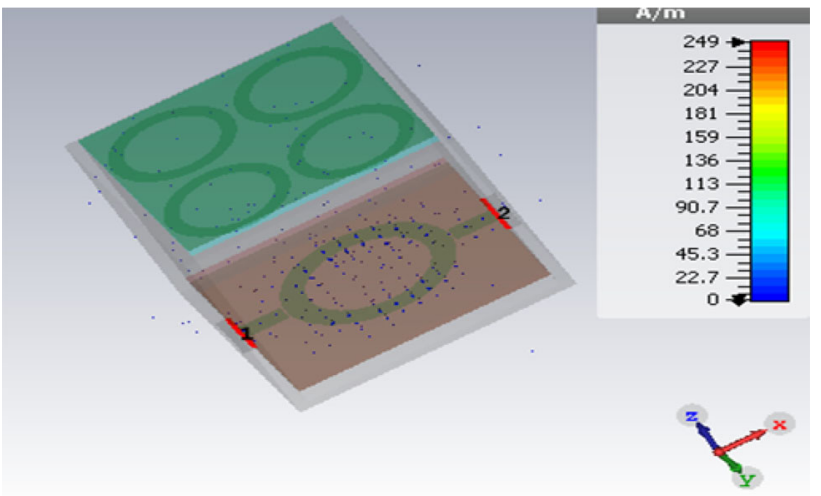

Figure 15. H Field for SMRR.

shows $\mathrm{Q}$ is directly proportional to capacitance $\mathrm{C}$ for parallel resonant circuit. Inductance $\mathrm{L}$ cannot increase as there is only EM coupling between lower and upper patch and not physical contact.

$$
\mathrm{Q}=\mathrm{R} \sqrt{\frac{\mathrm{C}}{\mathrm{L}}}
$$

Therefore, due to the dual resonance of SMRR, EM coupling increases which causes to enhance $\mathrm{E}$ field by increasing number of rings on parasitic patch. This reduces losses at resonating frequency and quality factor improves for SMRR with 4 rings.

The studies of Nishiyama [28] showed highly accurate near field analysis by FDTD method for stacked structure for variation in distance between two patches. SMRR simulated results reveal that the magnitude of E-field distribution on the parasitic patch increases as the distance hp increases above $0.3 \lambda$ and decreases after $0.7 \lambda$. Simulation results have shown that the resonating frequency and quality factor of the SMRR are the functions of the separation distance between the fed ring patch and parasitic ring patch. We have simulated the SMRR structure with the variation in hp from $0.3 \lambda$ to $0.7 \lambda$ to the optimized resonating frequency at $2.45 \mathrm{GHz}$ and maximum $\mathrm{Q}$. Thus, we mainly analysed the electromagnetic field and frequency response of the SMRR structure and found that electromagnetic coupling between the fed ring and parasitic ring is maximum at $\mathrm{hp}=0.45 \lambda$.

\subsection{Discussion on impedance bandwidth of SMRR}

Resonator technique keeps evolving to meet application requirement. To measure complex permittivity with resonator sensor return loss should be minimum and narrow bandwidth is desired. Kang et al [29] suggested the structural modification for improvement in impedance bandwidth with parasitic rings. It is noted that impedance bandwidth is widest around the natural resonant frequency. 
Table 8. Impedance bandwidth comparison for different resonator structures.

\begin{tabular}{lcccc}
\hline Resonator & Resonating frequency $(\mathrm{GHz})$ & $\mathrm{Z}_{11}(\mathrm{Ohms})$ & $\mathrm{S}_{21}(\mathrm{~dB})$ & Bandwidth $(\mathrm{MHz})$ \\
\hline Planar & 2.445 & 37.19 & -7.49 & 33.5 \\
Open boxed & 2.435 & 41.27 & -4.31 & 19.48 \\
SMRR (2 rings) & 2.458 & 42.65 & -3.83 & 25 \\
SMRR (3 rings) & 2.459 & 43.60 & -2.95 & 19 \\
SMRR (4 rings) & 2.443 & 44.54 & -2.61 & 15.26 \\
\hline
\end{tabular}

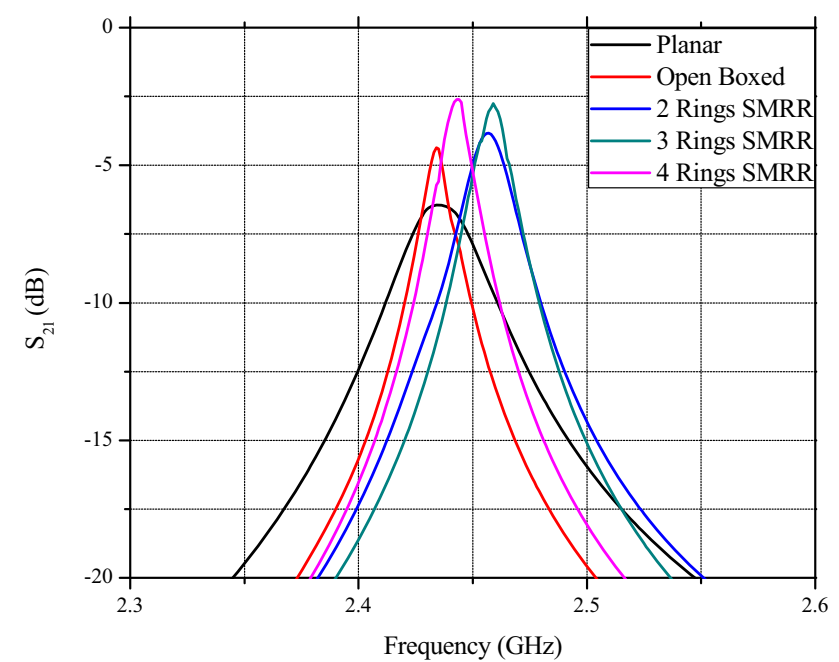

Figure 16. Variation in $S_{21}$ against frequency plot of various resonators.

When dielectric material is inserted, frequency shifts away from resonant frequency. Hoi et al [30] depicted that impedance bandwidth is dependent on energy loss of the system. For dielectric constant measurement application, maximum energy should be coupled to the output and loss in the system should be minimum. In planar microstrip ring resonator, we get wide bandwidth, which is not desirable in the said application. After simulation with different number of rings (table 8) showed that $Z_{11}$ increases as the number of rings increases. $Z_{11}$ is $44.54 \mathrm{Ohms}$ which is maximum for 4 rings SMRR. As number of rings increases bandwidth gets narrower for SMRR structure. Bafrooei et al [31] mentioned that impedance bandwidth can be controlled by parasitic elements. By experimentation and optimization of SMRR, it is noted that bandwidth goes on decreasing as losses are reduced by increasing number of rings on the parasitic patch. Figure 16 shows variations in $S_{21}$ against frequency of different resonators as mentioned in table 8 . This plot illustrates that bandwidth of 4 rings SMRR is reduced to half than the planar resonator. Resonator Sensor parameters like $S_{21}, Z_{11}$ and bandwidth are improved in optimised 4 rings SMRR.

SMRR analysis with respect to electromagnetic field and impedance bandwidth reveals that due to the dual resonance of SMRR amplitude of electric field between patches becomes large. The electric field is more due to capacitive coupling as number of rings increases on the parasitic patch. Impedance bandwidth can be controlled due to parasitic rings as required.

\section{Validation}

The measured permittivity values are evaluated against values described by standard data base and other published data [32-34]. Measured values are within 2\% range of calculated Q and frequency range. Table 9 showed average values of ten samples of various liquid complex permittivity values experimentally and compared with the database. The present technique is very significant when compared with the results published by Hippel [35], Verma [26] and Speight [34] for dielectric parameters. Other methods report accuracy of \pm 0.1 to \pm 0.39 for dielectric cover [19] and accuracy of 0.016 is reported [4]. Relative permittivity calculations using SMRR of specific

Table 9. Relative permittivity and loss tangent of test sample.

\begin{tabular}{|c|c|c|c|c|c|c|}
\hline \multirow[b]{2}{*}{ Test sample } & \multicolumn{2}{|c|}{ Experimentation } & \multirow[b]{2}{*}{ Standard Value $\varepsilon_{\mathrm{r}}$} & \multirow{2}{*}{$\begin{array}{l}\text { Exp. } \\
\tan \delta\end{array}$} & \multirow[b]{2}{*}{ Standard $\tan \delta$} & \multirow[b]{2}{*}{ Reference } \\
\hline & Mean $\varepsilon_{\mathrm{r}}$ & Deviation & & & & \\
\hline N-hexane & 1.895 & 0.003 & 1.89 & 0.00536 & 0.004 & {$[34]$} \\
\hline Wax & 1.939 & 0.003 & 1.93 & 0.00519 & 0.0072 & [26] \\
\hline Diesel & 2.016 & 0.005 & 2.02 & 0.00884 & 0.006 & [34] \\
\hline Kerosene & 2.097 & 0.003 & 2.09 & 0.00830 & 0.0045 & [26] \\
\hline Crude oil & 2.183 & 0.003 & 2.19 & 0.00705 & - & {$[32]$} \\
\hline Lubricant oil & 2.219 & 0.004 & 2.22 & 0.00917 & 0.009 & [35] \\
\hline
\end{tabular}


dimensions is observed \pm 0.1 to $\pm 0.4 \%$ accuracy. Loss tangent deviation is around \pm 0.0025 .

\section{Conclusion}

Stacked multi-ring resonator structure can be used for dielectric characterization of material like liquid and paste. In the used SMRR due to dual resonance, electromagnetic field coupling increases which causes the increase in quality factor and the decrease in loss. Losses are reduced for stacked ring resonator structure than planar resonator structure. Losses are further reduced by covering the box with different number of rings on the parasitic patch. Best results are obtained with four rings on the parasitic patch. As $Q$ is highest with SMRR among various resonator structures, the sensor is more accurate. Enhanced gain causes to improve sensitivity and stability of SMRR. Relative permittivity calculations are carried out with $0.1 \%$ accuracy. Electric field is increased in SMRR more than double compared to planar resonator. Total attenuation is reduced by five times than planar ring resonator system. Narrow bandwidth is achieved around $15.2 \mathrm{MHz} . \mathrm{Z}_{11}$ is increased to $44.75 \mathrm{Ohms}$. Sensitivity is achieved around $15.02 \mathrm{MHz}$ per $1 \%$ change in permittivity. Observed accuracy is \pm 0.1 to $\pm 0.4 \%$ for relative permittivity and \pm 0.00025 deviation for loss tangent.

The results of this model show good agreement with those generated by hexahedral TLM mesh using CST Studio software. The computed results are compared with experimental data, and again good agreement is obtained. As loss value in the liquid and paste sample is known, adulteration can be detected based on the results of experimental research in future.

\section{Acknowledgements}

The authors are indebted to Dr. Mahesh Abegaonkar, Centre for Applied Research in Electronics, IIT Delhi, India. We gratefully acknowledge the late Dr. Mrs. Savita Kulkarni, Maharashtra Institute of Technology, Pune, India for her technical guidance and Maharashtra Institute of Technology, Pune, India for sharing lab equipment.

\section{References}

[1] Venkatesh M S and Raghavan S S V 2005 An overview of dielectric properties measuring techniques. Canadian Biosystems Engineering 47: 15-30

[2] Chen L F, Ong C K and Neo C P 2004 Microwave Electronics: Measurement and materials characterization, John Wiley \& Sons, West Sussex, England, pp 37-119

[3] Kato Y, Horibe M, Ameya M, and Kurokawa S 2014 Study on measurement method of permittivity for low-loss materials using microstrip ring-resonator. In: Proceedings of Asia Pacific Microwave Conference, pp. 280-282

[4] Joshi K K, Pollard R D and Postoyalko V 1994 Variational analysis of microstrip with dielectric overlay and validation. IEE Proc. Microwaves, Antennas and Propagation 141(2): 138-140

[5] Kulkarni S G and Joshi M S 2015 Design and analysis of shielded vertically stacked ring resonator as complex permittivity sensor for petroleum oils. IEEE Transactions on MTT 63(8): 2411-2417

[6] Edwards T C 2000 Foundations of interconnect and microstrip circuit design. $3^{\text {rd }}$ edition, John Wily \& Sons Ltd, England, pp 84-128

[7] Bedair S S and Sobhy M I 1980 Accurate formulas for computer-aided design of shielded microstrip circuits. $\mathrm{Mi}$ crowaves, Optics and Antennas, IEE Proceedings H 127(6): 305-308

[8] Chang K and Hsiel L 2004 Microwave ring circuits and related structures. $2^{\text {nd }}$ edition, John Wiley \& Sons, New Jersey, pp 5-80

[9] Nishiyama E, Aikawa M and Egashira S 2004 Stacked microstrip antenna for wideband and high gain. IEE Proceedings Microwave Antennas Propagation, 151(2): 143-149

[10] Kunte A A, Gaikwad A N and Kulkarni S G 2015 Investigation of transmission line modelling method for dielectric characterization using ring resonator. In: IEEE International Applied Electromagnetic Conference AEMC, pp. 1-2

[11] Kishk A A, Xiao Zhang, Glisson A W and Kajfez D 2003 Numerical analysis of stacked dielectric resonator antennas excited by a coaxial probe for wideband applications. IEEE Transactions on Antennas and Propagation, 51(8): 1996-2006

[12] Kunte A and Kulkarni S 2008 Experimental investigation of complex permittivity and determination of ethanol content in gasoline. In: Proc IEEE International Microwave Conference, pp. 171-174

[13] Bernard P A and Gautray J M 1991 Measurement of dielectric constant using a microstrip ring resonator. IEEE Transactions on Microwave Theory and Techniques, 39(3): 592-594

[14] Egorov V N 2007 Resonance methods for microwave studies of dielectrics (review). Springer Instruments and Experimental Techniques, 50(2):143-175

[15] Heinola J M and Tolsa K 2006 Dielectric characterization of printed wiring board materials using ring resonator techniques: A comparison of calculation models. IEEE Transactions on Dielectrics and Electrical Insulation, 13(4): 717-726

[16] Abegaonkar M P, Karekar R N and Aiyer R C 2002 Perturbation of microstrip ring structure by grain moisture and its angle dependence. Microwave and Optical Technology Letters, 33(2): 128-132

[17] Hopkins R and Free C 2008 Equivalent circuit for the microstrip ring resonator suitable for broadband materials characterisation. IET Microwaves, Antennas and Propagation, 2(1): 66-73

[18] Rueggeberg W 1971 Arbitrarily dimensioned dielectric modules at microwave frequencies. IEEE Transactions on MTT, 19(6): 517-521

[19] Bahl I and Stuchly S 1980 Analysis of a microstip covered with a lossy dielectric. IEEE Transactions on Microwave Theory and Techniques, 28: 104-109 
[20] Ivanov S A and Peshlov V N 2003 Ring resonator method Effective procedure for investigation of microstrip line. IEEE Microwave and Wireless Components Letters, 13(6): 244-246

[21] Sarabandi K 1997 Microstrip ring resonator for soil moisture measurements. IEEE Transactions on Geoscience and Remote Sensing, 35: 1223-1231

[22] Svaina J 1992 Analysis of multilayer microstrip lines by a conformal mapping method. IEEE Transactions Microwave Theory and Techniques, 40: 769-772

[23] Kobayashi M 1988 A dispersion formula satisfying recent requirements in microstrip CAD. IEEE Transactions on Microwave Theory and Techniques 36(8): 1246-1250

[24] Kirschning M, Jansen R H and Koster N H L 1983 Measurement and computer-aided modeling of microstrip discontinuities by an improved resonator method. IEEE MTT-S International Microwave Symposium Digest 495-497

[25] Darko Kajfez 1984 Q factor measurements with network analyzer. IEEE Transactions on Microwave Theory and Techniques, 32(7): 666-670

[26] Verma A and Omar A 2005 Microstrip resonator sensors for determination of complex permittivity of materials in sheet, liquid and paste IEEE Proc. Microwave Antennas Propagation 152(1): 47-54

[27] Kajfez D 1999 Uncertainty analysis of the transmission type measurement of q factor. IEEE Transactions on Microwave Theory and Techniques, 47: 367-371
[28] Nishiyama E and Aikawa M 2001 Fdtd analysis of stacked microstrip antenna with high gain. Progress in Electromagentics Research 33: 29-43

[29] Kang K K and Lee J W 2007 An improved impedance bandwidth of modified UWB antenna with staircased parasitic rings. IEEE Antennas and Wireless Propagation Letters 6: $521-524$

[30] Ng Hoi K and Leung K W 2006 Frequency tuning of the linearly and circularly polarized dielectric resonator antennas using multiple parasitic strips. IEEE Transactions on Antennas and Propagation, 54(1):225-230

[31] Bafrooei P M and Shafai L 1999 Characteristics of single and double layer microstrip square ring antennas. IEEE Transactions on Microwave Theory and Techniques, 47(10):1633-1639

[32] Joshi K K and Pollard R D 1999 Microstrip resonator technique for non-destructive moisture/permittivity measurement. In: Proc. IEEE MTT International Microwave Symposium, 4: 1863-1866

[33] Joshi K K and Pollard R D 2006 Sensitivity analysis and experimental investigation of microstrip resonator technique for the in-process moisture/permittivity measurement of petrochemicals and emulsions of crude oil and water. IEEE MTT-S International Microwave Symposium Digest, pp. $1634-1637$

[34] Speight J G 2001 Handbook of Petroleum Analysis. Jones Wiley and Sons, New Jersey, pp 105-133

[35] Hippel A 1995 Dielectric materials and application. $2^{\text {nd }}$ edition, Artech House, USA, pp 362-367 